\title{
Les enjeux socio-environnementaux du développement durable en Amérique du Sud. Considérations à partir du cas colombien
}

The Socio-Environmental Issues of Sustainable Development in South America. Considerations Stemming From the Colombian Case

\section{Oscar Navarro}

\section{OpenEdition \\ Journals}

Édition électronique

URL : https://journals.openedition.org/developpementdurable/9045

DOI : 10.4000/developpementdurable.9045

ISSN : $1772-9971$

Éditeur

Association DD\&T

\section{Référence électronique}

Oscar Navarro, «Les enjeux socio-environnementaux du développement durable en Amérique du Sud. Considérations à partir du cas colombien », Développement durable et territoires [En ligne], Vol. 2, n 3 I Décembre 2011, mis en ligne le 02 décembre 2011, consulté le 21 septembre 2021. URL : http:// journals.openedition.org/developpementdurable/9045; DOI : https://doi.org/10.4000/ developpementdurable.9045

Ce document a été généré automatiquement le 21 septembre 2021.

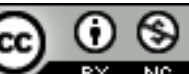

Développement Durable et Territoires est mis à disposition selon les termes de la licence Creative Commons Attribution - Pas d'Utilisation Commerciale 4.0 International. 


\title{
Les enjeux socio-environnementaux du développement durable en Amérique du Sud. Considérations à partir du cas colombien
}

\author{
The Socio-Environmental Issues of Sustainable Development in South America. \\ Considerations Stemming From the Colombian Case
}

Oscar Navarro

1 Le développement durable est communément pensé comme une solution aux problèmes écologiques, et est communément employé comme synonyme de protection de l'environnement. Cependant, l'interprétation polysémique du concept a conduit certains acteurs à produire de nouvelles alternatives au développement durable ${ }^{1}$. La question du développement durable se pose de façon tout aussi capitale dans les pays de l'Amérique Latine que dans tant d'autres pays du monde, et notamment ceux dits "développés ». Cependant, les interrogations et les propositions politiques se construisent à partir de certaines questions spécifiques. Ainsi, le développement durable se construit dans ce territoire à partir des problèmes sociaux et politiques, plus que sur les questions environnementales.

2 La Colombie permettra d'illustrer cette hypothèse, en montrant, autant que possible, la particularité de cette région du monde. Quatre pistes problématiques vont se succéder : (1) Nous observons qu'il existe une absence de consensus sur le sens attribué au terme "développement durable " entre les acteurs sociaux, y compris les chercheurs en sciences sociales. (2) Ce manque de consensus est dû, entre autres, à l'existence de modèles de développement structuralement différents, c'est-à-dire des visions distinctes du monde et de la société (écodéveloppement, ethno-développement, etc.). (3) Nous partons du constat d'une grande complexité sociale et politique propres à la région contexte de la réflexion (l'Amérique Latine) et plus particulièrement le cas de la 
Colombie, marquée par des inégalités majeures, la violence et la corruption politique, bref, l'instabilité institutionnelle, donnant des caractéristiques particulières aux enjeux écologiques et économiques émergents. (4) Dans ce contexte, nous considérons l'intérêt de placer la notion de Conflit (de l'analyse des bases et des dynamiques des conflits à la gestion de ceux-ci) au centre de la question afin d'identifier les obstacles qui entravent le développement durable. Saisir la spécificité du DD sur ce territoire suppose donc de comprendre les tensions que son utilisation révèle. Deux situations de conflits locaux nous permettrons d'illustrer cette tension : comment des zones de conservation (le parc naturel Katios et la Sierra Nevada de Santa Marta) mettent en avant la tension entre la volonté de conserver l'environnement et de gérer le développement des populations locales.

\section{Contexte latino-américain du Développement Durable}

3 Le paradigme du développement durable est fondé sur l'idée qu'il doit exister un équilibre entre le progrès économique des sociétés, la justice sociale et la préservation de l'environnement et des ressources nécessaires à la vie. Mais cet équilibre trouve différentes interprétations et applications, en fonction des contextes socio-historiques et les modalités de son appropriation par les acteurs concernés.

\subsection{Polysémie et confusion dans le contexte latino-américain}

4 Le terme fait l'objet de différentes interprétations (définition de son sens) ainsi que diverses modalités d'usage. Il est possible que l'utilisation d'un terme soit adopté de manière à déguiser les principes faisant allusion à son origine et à dissimuler des intentions contraires à ces mêmes principes de protection ou usage rationnel des ressources. Et c'est ce qu'il se passe dans des contextes institutionnels faibles ou corrompus de certains pays en voie de développement. Le terme est devenu une sorte de « marque », d'image employée par les gouvernements, les organisations et les entreprises (Palacio et al. 2009). On peut ainsi s'interroger sur le décalage entre la valorisation d'une pratique singulière (les monocultures de palmier à huile pour la production d'agrocombustibles en Colombie), qui font pourtant l'objet de distinctions internationales, alors même qu'elles ne sont pas sans conséquences environnementales et sociales (Mingorance, 2007).

5 En Amérique Latine, les termes «soutenable» ou «durable» sont utilisés indistinctement, sous prétexte qu'ils sont synonymes. Pourtant, l'utilisation de l'un ou de l'autre peut constituer deux formes de réalité. Parler de Desarrollo sostenido (développement soutenu), indique que le développement économique doit continuer avec le même rythme et les mêmes caractéristiques actuelles. Le mot perdurable fait allusion à l'effort que nous devons faire pour garantir des ressources dans le futur pour nos enfants sans changer le modèle économique et de confort social. Il y a là deux acceptations du terme qui se basent sur l'idée de payer le prix du modèle actuel sans le revoir dans le fond, en oubliant l'inspiration de cette démarche qui est le constat de la «limite de la croissance » (Meadows, 1973). Ces mots sont utilisés notamment par les gouvernements ou par les entreprises, mais aussi par certains organismes internationaux et même par des chercheurs. Le terme sostenible (durable) plus largement utilisé notamment par les 
écologistes et les intellectuels ferait allusion au besoin de revoir de façon critique l'actuel modèle de développement et là les nuances se multiplient.

\subsection{Développement Durable : des visions différentes du développement en Amérique Latine}

6 Le choix de ces adjectifs est bien évidemment en relation à la conception que l'on a du développement lui-même. C'est-à-dire 'univers idéologique autour de l'objet qui exprime une vision du monde et de la société, fondée sur des rationalités distinctes, voire en concurrence ou en opposition.

7 Dans ce sens, nous trouvons par exemple le paradigme de l'écodéveloppement ( ecodesarrollo) appelé aussi du développement vert, qui est antérieur à celui de développement durable. Il a été proposé durant les années 1970 par Strong et Sachs (Sachs, 1980,1982) qui suggéraient un modèle de développement basé sur une théorie économique renouvelée avec des considérations écologiques. Ce modèle propose de faire emphase ou de donner une priorité à la durabilité environnementale par rapport à la dimension économique et sociale. Ce modèle prend de l'importance en Amérique Latine accompagné par les mouvements altermondialistes et ethno-culturalistes.

Dans ce sens il s'est configuré plus récemment un autre terme, celui de "Ethnodéveloppement", qui se base sur l'idée de la reconnaissance des autres modèles de développement existants, différents du modèle occidental. Un exemple précis de cette démarche est la reconnaissance des modèles de développement des peuples amérindiens, réputés plus viables écologiquement parlant, et ayant gagné une légitimité sociale, voire politique et juridique dans certains pays sud-américains comme la Bolivie par exemple (Albo, 1996). Le principe fondateur est basé sur le développement en tant que « processus autonome que représente la vision d'une communauté, de son histoire, ses valeurs et ses buts pour atteindre une meilleure qualité de vie» (Bonfil, 1982). Cette tendance a été soutenue indirectement par l'ONU lors de la Déclaration du respect à la diversité culturelle (signée le 2 novembre 2001), reconnue comme étant aussi importante pour l'homme que la diversité biologique. Certains mouvements écologistes radicaux se servent également de ce courant pour argumenter leurs propositions. Dans tous les cas, les principes inspirateurs de celui-ci sont le protectionnisme (conservation) du patrimoine naturel, l'usage rationnel et/ou équilibré des ressources, la performance technique, la diminution de la pression sur les écosystèmes fragiles, etc.

\section{Développement durable ou enjeux socio- environnementaux?}

9 Les débats et les divergences autour de la question du développement durable révèlent notamment l'existence des tensions, voire des conflits socio-environnementaux. On constate que les effets néfastes sur l'environnement et les écosystèmes sont dus, en bonne partie, aux déséquilibres existants à l'intérieur même des sociétés humaines, à l'inégale répartition des ressources et à la difficulté d'y accéder de la part de certaines catégories de populations, aux différentes conceptions du développement et des rapports à l'environnement ainsi qu'aux différents modes de vie et de conceptions du bien-être. Les activités humaines engendrent, par définition ou par nature, des conflits du fait de la 
compétition pour l'accès et l'usage des ressources nécessaires à la vie et au développement des sociétés. C'est pourquoi il est important de comprendre ces tensions à partir de l'analyse des bases et des dynamiques sociales sous-jacentes.

\subsection{Conflits d'accès et d'usage du territoire et/ou de ses ressources}

La notion de conflit évoque, de façon générale, une lutte faisant appel à des actions allant du débat policé à la confrontation violente, entre protagonistes conscients de l'incompatibilité de leurs positions, mettant en cause des intérêts et des valeurs. Les phénomènes de périurbanisation, les nuisances des installations industrielles, des infrastructures de transport ou de production d'énergie, l'étalement résidentiel, les pollutions d'origine agricole, sont autant de manifestations de tensions et de conflits portant sur les usages des territoires (Kirat et Torre, 2008). Les travaux menés sur la question ont montré que les conflits d'usage des espaces et des ressources naturelles sont expliqués, entre autres, par la multiplication des confrontations entre les rationalités différentes des acteurs impliqués (individus, groupes ou organisations). Il est donc nécessaire d'essayer de mieux comprendre les bases et les dynamiques sociocognitives des conflits autour de la question environnementale afin d'identifier et expliquer les obstacles d'un développement harmonieux des sociétés. Une telle perspective peut prendre deux directions: 1) l'étude de la base de la connaissance sociale dans l'émergence, la gestion et la solution des conflits (représentations sociales, croyances, idéologies) ; 2) l'analyse psychosociale de la dynamique des conflits : le rôle des identités sociales et du rapport au territoire dans la compréhension et la gestion des conflits. Analyse des pratiques et des évaluations (jugements) entre les acteurs confrontés.

\subsection{La Colombie : un exemple de contexte difficile pour le développement durable}

11 Dans les pays en développement tels que la Colombie ou ceux de l'Amérique Latine en général, la question de la justice sociale et économique prend le pas sur la question environnementale.

\section{Une richesse écologique menacée}

La Colombie, située à l'extrême nord de l'Amérique du Sud, est un pays jouissant d'une diversité biologique et culturelle très importante, de par sa localisation tropicale et son histoire faite de mélanges culturels. Elle possède $10 \%$ de la diversité biologique mondiale alors que son territoire de 1,14 millions de $\mathrm{km}^{2}$, ne représente que $0,75 \%$ de la surface de la planète (pays possédant l'un des 34 points chauds de la biodiversité mondiale). 
Tableau 1 : Pertes de milieux naturel dans les pays d'importance majeure pour la biodiversité

\begin{tabular}{|c|c|c|c|}
\hline \multirow{2}{*}{ Pays } & \multicolumn{2}{|c|}{$\begin{array}{c}\text { Densité de } \\
\text { population (par } \\
\mathbf{k m 2} \text { ) }\end{array}$} & $\begin{array}{c}\text { Perte de milieux historiquement associée } \\
\text { avec la densité de population projetée pour } \\
\mathbf{2 0 5 0}(\%) \text { un total en moyenne de 70\% }\end{array}$ \\
\cline { 2 - 3 } & 1995 & 2050 & \\
\hline Brésil & 188 & 288 & 41 \\
\hline Madagascar & 256 & 874 & 67 \\
\hline Mexique & 478 & 807 & 67 \\
\hline RD Congo & 200 & 726 & 67 \\
\hline Colombie & $\mathbf{3 4 5}$ & $\mathbf{6 0 0}$ & $\mathbf{7 8}$ \\
\hline Indonésie & 1090 & 1757 & 85 \\
\hline
\end{tabular}

(Broswimmer, 2003)

13 Cependant, la déforestation et la dégradation des écosystèmes sont une menace pour les espèces et ceci s'explique par différents facteurs, dont les conditions d'accès aux ressources, l'absence de mécanisme de contrôle et de sanction pour les dommages causés aux écosystèmes, la situation socioéconomique d'une grande partie de la population qui l'oblige à exploiter les ressources naturelles sans les moyens techniques adaptés, les processus d'occupations non planifiées, et enfin, le manque de recherche sur le sujet engendrant une base scientifique ainsi qu'un système d'information insuffisant.

Cette gestion institutionnelle étant inefficace on doit chercher ses racines dans les problèmes sociaux et politiques du pays marqué par la violence, l'anarchie et la corruption institutionnelle. Une grande richesse écologique se détériore à cause de l'absence de gestion et du contrôle des autorités environnementales. Le paradoxe est qu'au cours des dernières années en Colombie les indicateurs économiques ont présenté une croissance considérable, situation qui ne se voit pas reflétée dans la réalité sociale du pays.

\section{Conflit social et politique accru : violence et absence institutionnelle}

La Colombie est réputée pour son conflit social et politique qui dure depuis plus de 50 ans. Ce conflit a connu différentes phases, mobilisant différents acteurs, des groupes de guérillas paysannes et communistes, en passant par les armées privées paramilitaires et mafieuses, et, face à eux, un État qui se révèle impuissant à maîtriser la violence du pays, faute d'une armée suffisamment puissante et en raison, aussi, de ses difficultés à imposer une ligne politique.

Jusqu'à présent, le personnel dirigeant colombien n'a pas su différencier ses intérêts privés de ceux de l'État, ni attribuer une place claire aux intérêts économiques étrangers dans la politique intérieure. Il ne peut donc pas apparaître comme le représentant des intérêts de tous les groupes sociaux. L'État n'a pas su contenir la violence exercée par différents groupes armés sur la population civile. Certaines catégories sociales (notamment les paysans et les minorités ethniques habitant en zone rurale, voire dans des zones écologiquement stratégiques, des zones de conservation) estiment avoir été abandonnées par cet État. Le climat institutionnel n'est donc pas propice à prendre en charge des enjeux strictement environnementaux. Il est nécessaire de mentionner également le problème de production et de trafic de drogue, qui est un facteur de complexification du conflit. Les cultures de coca et de pavot ont augmenté de manière 
vertigineuse ces dernières années, malgré la "guerre » déclarée il y a quelques années par le gouvernement avec le soutien décidé des États-Unis et qui s'est avérée inefficace.

Dans ce cadre institutionnel et social si complexe - où les problèmes sociaux tels la pauvreté, la corruption, la menace des groupes armés illégaux, dominent -, les questions environnementales ne sont pas prioritaires. Les dimensions constituant le développement durable sont en déséquilibre et c'est la protection de l'environnement qui est sacrifiée. Cela peut, en partie, expliquer la difficulté d'instituer un Ministère de l'Environnement disposant de réelles compétences. Celui-ci est crée tardivement, en 1993, suite à la conférence de Rio. Depuis, il a changé de nombreuses fois de dénomination (actuellement, celui-ci se nomme Ministère de l'environnement, du logement et du développement territorial), et ses attributions sont réduites. Il s'occupe surtout de la prestation de services publics liés à l'habitat et son rôle de garant de la protection environnementale n'est relégué qu'à un service de délivrance de permis d'exploitation de ressources naturelles. De ce fait, la politique de l'État colombien en matière environnementale demeure très limitée. Par ailleurs, dans ce domaine aussi, la corruption s'installe; la presse s'est fait l'écho de nombreux cas litigieux d'attribution de permis d'exploitation de zones de conservation qui violent la législation en vigueur (Palacio et al. 2009). C'est le cas de l'autorisation donnée par l'État à une multinationale sud-africaine pour l'exploitation d'une éventuelle mine d'or dans une région au centre du pays disposant d'un parc naturel. Ceci fait partie de la politique de confiance accordée aux investisseurs lancée par le gouvernement actuel, même si elle met en péril la politique de conservation.

Un autre exemple est le fait que le gouvernement colombien se lance dans un développement massif de plantations de palmiers à huile, de canne à sucre et d'autres monocultures pour la production d'agro-carburants, au détriment des forêts tropicales humides, de la biodiversité et du respect des droits de l'homme. En effet, des centaines de milliers d'hectares sont transformés et le développement de cette monoculture va de pair avec la destruction à grande échelle des forêts tropicales humides qui s'accompagne de graves violences et de violations des droits de l'homme. On connait les actions des groupes paramilitaires qui ont chassé les paysans de leurs terres en perpétrant de nombreux massacres, produisant ainsi des déplacements importants de populations. L'appropriation illégale de ces terres a ensuite été légalisée par le gouvernement lors du processus de paix avec ces groupes paramilitaires.

19 Cependant, la société civile reste active et organisée pour faire face à ces actes. Le " referendum pour l'eau $»^{2}$ en est un exemple. Il s'agit d'une initiative populaire qui a été proposée au Congrès de la République pour que l'accès à l'eau soit déclaré dans la Constitution en tant que droit fondamental, ce qui oblige ainsi l'État à en garantir l'accès pour tous les Colombiens. Cette initiative de réforme constitutionnelle est actuellement amendée puisque certains congressistes ont essayé de changer l'esprit de la proposition en enlevant le caractère public de sa gestion, ouvrant ainsi la porte à sa privatisation.

Un autre exemple est la déclaration d'inconstitutionnalité de la Loi de Développement Rural et Forestière (février de 2003) élaborée par le gouvernement. En effet, le Conseil Constitutionnel a confirmé que les consultations préalables nécessaires n'avaient pas été réalisées, notamment auprès des communautés indiennes et d'afro-descendants. 


\section{Exemples des conflits socio-environnementaux en Colombie} de la Biosphère en 1989, où cohabitent des catégories d'usagers diverses (industriels, paysans agriculteurs, indiens, entre autres). Dans cette région se révèlent des paradoxes et contradictions liés à l'usage de l'eau, propres au contexte latino-américain. Des conflits permanents autour de l'eau se développent, motivés principalement par la construction d'ouvrages de grande envergure pour la maîtrise de l'eau, prévue au milieu d'une zone de conservation (le parc naturel et une réserve indienne), engendrant l'opposition de différents acteurs politiques, associatifs et des riverains ainsi que de nombreuses confrontations dans un climat de grande tension du fait de la présence des groupes armés illégaux dans cette zone, soutenant par ailleurs la construction de tels ouvrages.

même, la côte pacifique colombienne qui est la région la plus riche en ressources minières et forestières du pays est également la plus pauvre économiquement, se trouvant fortement touchée par le conflit armé interne. C'est dans cette région que se situe le parc national naturel Katios, possédant l'une des plus grandes diversité biologique du pays, une importante forêt tropicale où s'opposent des intérêts divers et donc des conflits entre l'État colombien, les communautés indigènes, les investisseurs et entreprises internationales qui souhaitent la construction d'une grande autoroute internationale (la Panamericana), liant l'Amérique du Sud avec l'Amérique Centrale et qui détruirait le parc, la biodiversité et les formes culturelles ancestrales d'appropriation du territoire.

régions qui présentent des conflits similaires où se confrontent des conceptions culturelles différentes de la nature, de l'environnement et du territoire de par la présence des divers groupes culturels et des catégories d'acteurs confrontées. Des zones de conservation qui sont la cible de grands ouvrages et des enjeux économiques qui menacent non seulement la diversité biologique, mais également des populations y habitant bien avant l'arrivée des Européens sur le continent et qui violent des accords internationaux en matière de protection environnementale, dont la Colombie est cosignataire.

\section{Enjeux socio-environnementaux autour de l'eau dans la Sierra Nevada de Santa Marta}

L'analyse des différences culturelles autour de la ressource dans cette région de Colombie, nous offre un cadre interprétatif pour mieux comprendre la base de ces tensions. D'un côté, nous avons l'existence des représentations sociales différentes de l'eau parmi les usagers de la même source d'eau (même bassin versant), distingués par leur cadre de vie et leur appartenance culturelle. Ensuite, les différents jugements et évaluations entre catégories d'usagers nous donnent une idée de la dynamique du conflit et de la perception que ces usagers ont de leurs rivaux pour ce qui est de l'usage de l'eau.

Développement durable et territoires, Vol. 2, n 3 | Décembre 2011 


\section{Représentations sociales (RS) de l'eau}

fondamentalement par la distinction de valeurs identitaires, mais aussi par la différence de pratiques liées à la source. En effet, et malgré l'existence d'éléments communs dans la RS chez les trois groupes (représentation collective, universelle de l'eau), nous avons constaté l'existence d'au moins deux RS de l'eau: une RS «utilitariste» ou fortement marquée par une vision fonctionnelle et économique de l'eau, ainsi que par une perception instrumentalisée du cycle de l'eau (eau domestique), propre aux citadins et à la plupart des paysans. Deuxièmement, une RS «écologique» qui intègre l'eau au territoire ou à la nature elle-même, marquée par une perception non-instrumentalisée de la source (eau naturelle) exprimant une préoccupation pour son état, fondamentalement propre aux indiens et à une minorité des paysans. Cette différence des RS est due à l'influence du cadre de vie (rural ou urbain) ainsi qu'au cadre socioculturel (citadin, paysan ou indien), c'est-à-dire au système idéologique du groupe d'appartenance (Navarro 2009). 
Graphique 1 : Graphes de similitude. RS de l'eau par groupe d'usagers de l'eau de la SNSM

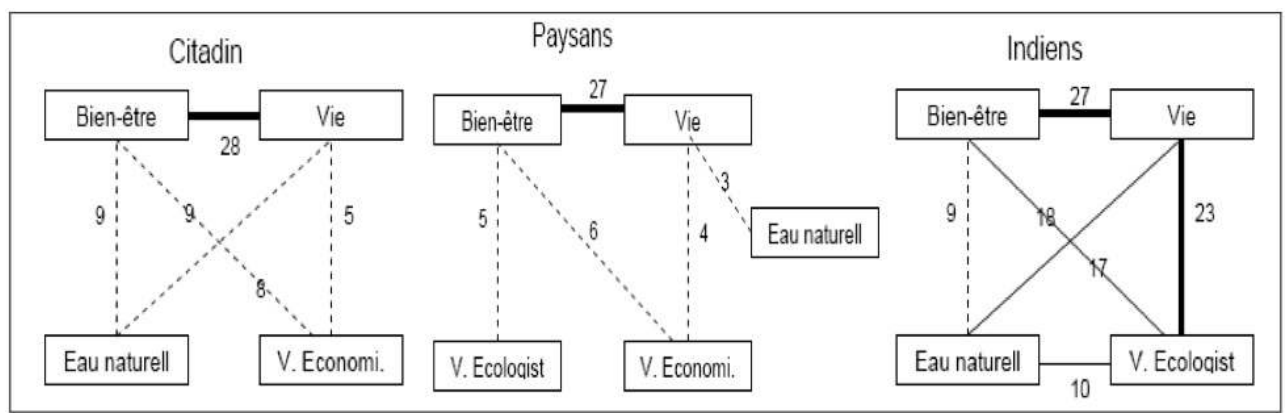

Évaluations et jugements entre catégories d'usagers de la même ressource en eau ressource est explicative de conflits qui s'attisent par les usages et l'accès à celle-ci (Moser et al. 2010). Nous avons voulu aborder les éléments subjectifs de la dynamique du conflit, c'est-à-dire les perceptions intergroupes et les attributions en relation aux valeurs et aux usages de la source. Ceci peut nous aider à comprendre comment les groupes se voient entre eux par rapport à l'eau ainsi que les attributions des responsabilités qu'ils font sur l'état actuel de la ressource.

Les sujets enquêtés ont créé une échelle entre les «bons » et les « mauvais » usagers de l'eau opposant les citadins aux ruraux, ce qui engendre d'importantes implications sur la façon dont les groupes attribuent des responsabilités par rapport à la dégradation de la source de la Sierra et les conséquences sur leur vie de manière générale. Les citadins étant donc considérés comme les "mauvais » usagers et les indiens comme les «bons » usagers, des regards critiques entre les groupes se sont élevés.

Tableau 2 : Perceptions, attributions et jugements intergroupes par rapport à l'eau dans la SNSM

\begin{tabular}{|l|c|c|c|c|}
\cline { 2 - 5 } \multicolumn{1}{c|}{} & \multicolumn{1}{c|}{$\begin{array}{c}\text { Attribution des } \\
\text { Significations de } \\
\text { l'eau }\end{array}$} & $\begin{array}{c}\text { Estimation de } \\
\text { quantité d'eau } \\
\text { utilisée }\end{array}$ & $\begin{array}{c}\text { Types } \\
\text { d'usages }\end{array}$ & $\begin{array}{c}\text { Jugement sur } \\
\text { l'usage }\end{array}$ \\
\hline $\begin{array}{l}\text { Citadins (selon les } \\
\text { indiens et les } \\
\text { paysans) }\end{array}$ & $\begin{array}{c}\text { Indispensable pour la vie } \\
\text { Valeur économique } \\
\text { Bien-être } \\
\text { L'eau est banalisée et } \\
\text { négligée }\end{array}$ & $\begin{array}{c}\text { Indiens : trop } \\
\text { Paysans : entre trop et } \\
\text { moins du nécessaire }\end{array}$ & $\begin{array}{c}\text { Taches } \\
\text { domestiques } \\
\text { Soin personnel } \\
\text { Industrie et } \\
\text { loisirs }\end{array}$ & Mauvais usage \\
\hline $\begin{array}{l}\text { Paysans (selon les } \\
\text { indiens et les } \\
\text { citadins) }\end{array}$ & $\begin{array}{c}\text { Valeur économique } \\
\text { Vitale pour leur existence } \\
\text { physique } \\
\text { Un outil de travail }\end{array}$ & $\begin{array}{c}\text { Entre peu et beaucoup } \\
\text { d'eau }\end{array}$ & $\begin{array}{c}\text { Activités } \\
\text { économiques } \\
\text { Taches } \\
\text { domestiques } \\
\text { Soin personnel. }\end{array}$ & $\begin{array}{c}\text { Plutôt un bon } \\
\text { usage }\end{array}$ \\
\hline $\begin{array}{l}\text { Indiens (selon les } \\
\text { paysans et les } \\
\text { citadins) }\end{array}$ & $\begin{array}{c}\text { Indispensable pour la vie } \\
\text { Est une divinité } \\
\text { Valeurs culturelles de } \\
\text { conservation }\end{array}$ & $\begin{array}{c}\text { Citadins : plus du } \\
\text { nécessaire } \\
\text { Paysans : entre peu et } \\
\text { moins du nécessaire }\end{array}$ & $\begin{array}{c}\text { économiques } \\
\text { Taches } \\
\text { domestiques } \\
\text { Rituels }\end{array}$ & Bon usage \\
\hline
\end{tabular}

31 Les jugements que l'on fait sur l'usage de l'eau chez les autres usagers d'une même source caractérisant les relations intergroupes sont en relation avec au moins trois aspects : tout d'abord les valeurs attribuées aux autres usagers en fonction de la source. Par exemple, les citadins sont vus comme de mauvais usagers, ceci étant en relation avec des valeurs qui leur sont attribuées: ils négligent et banalisent l'eau, engendrant de la sorte des irresponsabilités autour de son usage. On attribue généralement aux paysans des valeurs économiques et l'on souligne l'importance de la ressource pour leur subsistance et aux 
indiens des valeurs culturelles respectueuses de l'environnement. Deuxièmement, ces jugements des usages sont en relation avec la perception des types d'usages chez les autres catégories d'usagers. Ce qui veut dire que le problème n'est pas posé sur l'usage en tant que tel, mais sur la façon dont celui-ci est réalisé. Cette question nous amène au dernier élément en relation aux jugements sur les usages de l'eau : l'influence du cadre de vie. En effet, nous avons remarqué que les usagers habitant en milieu urbain sont toujours moins bien perçus en relation à l'usage de la source que ceux habitant en milieu rural. Cependant, ceux qui habitent en milieu urbain ont tendance à juger plutôt positivement l'usage que les habitants en milieu rural font de l'eau (Navarro, 1998). Finalement nous pouvons avancer l'hypothèse de l'influence qu'ont les représentations sociales sur les perceptions et les jugements entre catégories d'usagers partageant une même ressource en eau et l'intérêt que ce type d'informations a pour nous permettre de mieux comprendre les enjeux liés à la promotion d'un développement durable et plus particulièrement dans des pays en voie de développement.

\section{Conclusion}

Nous avons essayé d'illustrer, à travers le cas de la Colombie et des situations précises comme les conflits autour de l'eau dans la Sierra Nevada de Santa Marta, une préoccupation qui est de plus en plus grandissante : le fait d'accepter le paradigme du développement durable sans le confronter aux réalités, souvent difficiles, de contextes sociaux et institutionnels particuliers de certaines régions. De façon plus radicale, nous pensons qu'il est, dans certains cas, plus convenable d'aborder les problèmes sociaux et écologiques comme des enjeux socio-environnementaux, permettant ainsi de les concevoir de façon inductive, contextualisée et adaptée, et non prétendre les définir de façon générale et inadaptée. Il est clair que le développement durable constitue un cadre politique et économique, voire moral, pour la gestion des ressources et du développement. Mais il est vrai aussi qu'il peut cacher des désaccords, des tensions et des inconsistances dans les discours des responsables de la gestion des politiques du développement. Ceci est encore plus vrai dans des contextes politiques et institutionnels faibles, tel est le cas de la Colombie ou bien d'autres pays du monde. La difficulté à répondre aux principes du développement durable est plus liée aux problèmes endémiques de ces sociétés (corruption, violence) qu'aux difficultés techniques de son application.

Notre réflexion présente des limitations de type théorique du fait de devoir, de manière aussi succincte, aborder une question qui demande une multiplicité de lectures : sciences politiques, droit international, anthropologie, entre autres. Avec ces limitations et en partant d'un regard psychosociologique, nous avons tenté de participer à cette réflexion, en apportant des éléments nouveaux et concrets sur la façon de comprendre cette base sociocognitive des conflits, des tensions et des différences culturelles. C'est le cas de la théorie des représentations sociales qui nous laisse voir comment dès la base culturelle et environnementale qui constitue la vie des gens, des habitants, des usagers, les inconsistances et les tensions se forment. Ce niveau micro doit être pris en compte par le niveau d'analyse macro des ces questions propres au développement durable. L'applicabilité de ces principes, l'efficacité même de ces propositions, seront déterminées par cette base sociocognitive. Le contraire, c'est-à-dire la méconnaissance de cette base, nous amène à insister sur un regard naïf des institutions, sur les possibilités du 
développement durable et sa réelle capacité et vocation à résoudre les problèmes de la société contemporaine.

\section{BIBLIOGRAPHIE}

Albo X.,1996, Pobreza, Desarrollo, e Identidad Indígena. In Desarrollo Indígena : Pobreza, Democracia, Sustentabilidad. La Paz : Fondo Indígena y BID.

Bonfi G.,1982, El Etnodesarrollo : Sus Premisas Jurídicas, Políticas, y de Organización. In America Latina : Etnodesarrollo, Etnocidio. Costa Rica : Ediciones FLACSO.

Bouguerra M. L., 2003, Les batailles de l'eau. Pour un bien commun de l'humanité. Enjeux Planète. Paris.

Breslin P.,1986, "A Sense of Identity. Grassroots Development". The Journal of the InterAmerican Foundation. $10: 2: 12-21$.

Broswimmer F., 2003, Ecocide : une brève histoire de l'extinction en masse des espèces. Paris : Parangon Brundtland G.H., 1987, Our Common Future. Oxford : Oxford University Press

Kirat T. et Torre A. (Dir.), 2008, Territoires de conflits. Analyses des mutations de l'occupation de l'espace . Paris : éditions l'Harmattan.

Meadows, 1973, Halte à la croissance? Rapport sur les limites de la croissance. Paris : Edition Fayard.

Mingorance F., 2007, El flujo del aceite de palma Colombia - Bélgica/Europa.

Acercamiento desde una perspectiva de derechos humanos, in Lipietz A. Les arbres du mal. La production d'agro-carburant à partir du palmier à huile. Politis $\mathrm{n}^{\circ} 954$.

Moser G., Navarro O., Ratiu E. \& Weiss K., 2010, Relationships to water in the light of sustainable development. In V. Corral-Verdugo : Psychological Approaches to Sustainability. Nova Press.

Moser G., Weiss K, 2003, Espaces de vie. Aspects de la relation homme-environnement. Armand Colin, collection « Regards », Paris.

Navarro O., 2008, L'eau comme enjeu : territoire, identité et conflits d'usage in Kirat T. et Torre A. (Dir.) Territoires de conflits. Analyses des mutations de l'occupation de l'espace. Paris : éditions l'Harmattan.

Navarro O., 2009, Représentations sociales de l'eau dans un contexte de conflits d'usage : le cas de la sierra Nevada de santa Marta, Colombie. Cahiers Internationaux de Psychologie Sociale. $\mathrm{N}^{\circ} 81$ mars, pp. 65-86.

Palacio G. (Coord.), 2009, La manzana de la discordia. Bogotá : Ecofondo.

Sachs I., 1982, Ecodesarrollo : desarrollo sin destrucción. México : El Colegio de México.

Sachs I., 1980, Stratégies de l'écodéveloppement. Paris : éditions Économie et humanisme et les Éditions ouvrières. 


\section{NOTES}

1. Huitièmes journées d'études en psychologie sociale. Développement durable : question du sens. 18 et 19 juin 2009, Brest.

2. Signé par 2 millions de colombiens et présenté au parlement colombien en avril de 2009 .

\section{RÉSUMÉS}

Parler du développement durable dans le contexte des pays émergents tels ceux de l'Amérique Latine et particulièrement la Colombie, implique d'aborder le caractère conflictuel lié à la possibilité d'y atteindre les buts de ce paradigme du développement. Il est donc nécessaire de connaitre les obstacles sociopolitiques et culturels que rencontrent ces sociétés (corruption et instabilité institutionnelle, conflit armé, pauvreté, etc.). Étudier la base et les dynamiques psychosociales des conflits socio-environnementaux permet de mieux comprendre ces obstacles, mais aussi de dégager des réponses afin de mieux gérer ces tensions. Le cas de la Colombie et plus particulièrement des conflits autour de l'eau dans la Sierra Nevada de Santa Marta nous permettent d'illustrer nos propos.

Talk about sustained development in emergent countries such as in Latin America and specifically Colombia, implies to tackle the conflicting character linked to the possibility to aim the objectives of this development paradigm. It is so necessary to know socio-political and cultural obstacles these societies meet (corruption and institutional instability, armed conflict, poverty, etc...). Study socio-environmental conflict's bases and psychosocial dynamics help to better understand these obstacles, but also to find out answers to better manage this pressure. Colombia's case and water conflicts in the "Sierra de Santa Marta" above all will allow us to give an illustration of this matter.

\section{INDEX}

Mots-clés : Colombie, enjeux socio-environnementaux, représentation sociale de l'eau

Keywords : Colombia, social representation of water, socio-environmental stakes

\section{AUTEUR}

\section{OSCAR NAVARRO}

Oscar Navarro, Université de Antioquia, Colombie et UMR PACTE, Sciences Po Grenoble, est docteur en Psychologie spécialité Psychologie Sociale et Environnementale, ses intérêts de recherche tournent autour de l'analyse des conflits socio-environnementaux, particulièrement l'usage de l'eau et le partage du territoire. Ses dernières publications sont : Moser G. Navarro 0. Ratiu E. \& Weiss K. (2010) Relationships to water in the light of sustainable development. In V. Corral-Verdugo : Psychological Approaches to Sustainability. Nova Press : New York. Navarro, O. 
(2009) Représentations sociales de l'eau dans un contexte de conflits d'usage : le cas de la sierra Nevada de santa Marta, Colombie. Cahiers Internationaux de Psychologie Sociale. $\mathrm{N}^{\circ} 81$ mars,

pp. 65-86. 